\title{
Radial forearm and forehead flap reconstruction following resection of a nasal arteriovenous malformation: A case report
}

\author{
CHIH-SHIN LIN ${ }^{1}$, YUNG-SONG LIN ${ }^{1}$, BOR-SHYH LIN ${ }^{2}$, CHING-FENG LIEN ${ }^{3}$ and CHING-FENG LIU ${ }^{1,4}$ \\ ${ }^{1}$ Department of Otolaryngology, Chi-Mei Medical Center, Tainan 710; ${ }^{2}$ Institute of Imaging and Biomedical Photonics, \\ National Chiao Tung University, Tainan $711 ;{ }^{3}$ Department of Otolaryngology, E-DA Hospital and I-Shou University, \\ Kaohsiung 84001; ${ }^{4}$ Department of Biological Science and Technology, National Chiao Tung University, \\ Hsinchu, Taiwan 300, R.O.C.
}

Received March 26, 2015; Accepted April 22, 2016

DOI: $10.3892 / 01.2016 .5022$

\begin{abstract}
Arteriovenous malformation (AVM) is a structural vascular abnormality in which the arterial vasculature connects with the venous vasculature without capillary connections. AVM confined to the nasal cavity is considerably rare. Blurred vision can be one of the associated symptoms. A combined approach of complete surgical resection with prior superselective embolization is the treatment of choice. Following resection, the reconstruction of facial defects, particularly in the nasal area, is challenging. The present study reports the rare case of a patient with an AVM in the nasal cavity, in which embolization, resection and flap reconstruction were performed. Embolization and complete surgical resection were used to avoid recurrence. Subsequently, a combination of free radial forearm and forehead flaps was used for the reconstruction of the nasal defect, without prosthesis. To the best of our knowledge, this is the first report of AVM confined to the nasal cavity, managed by a combination of free radial forearm and forehead flap reconstruction following complete resection. The cosmetic results of the procedure were acceptable.
\end{abstract}

\section{Introduction}

Arteriovenous malformation (AVM) is an abnormal connection between arteries and veins that bypasses the capillary system. AVM lacks the dampening effect of capillaries on the blood flow, which means that the malformation can become progressively larger over time as the amount of blood flowing through it increases (1-3). An AVM confined to the nasal cavity is rare, and its high recurrence rate may be due to the use of embolization with incomplete or no resection $(2,4,5)$.

Correspondence to: Dr Ching-Feng Liu, Department of Otolaryngology, Chi-Mei Medical Center, 901 Chung Hwa Road, Yung Kung, Tainan 710, Taiwan, R.O.C.

E-mail: ellery51.holmes@gmail.com

Key words: arteriovenous malformation, nasal cavity, free flap, local flap
A combined approach of complete surgical resection with prior superselective embolization is the treatment of choice. Following resection, the reconstruct of facial defects is challenging, particularly in the nasal area (1). Only a few studies have reported the use of either local or free flaps alone for the reconstruction of AVMs involving the nasal cavity following resection (1-5); however, a combination of free and local flaps may be used to achieve an acceptable cosmetic result. The present study reports a case of nasal reconstruction using a free radial forearm flap in combination with a forehead flap, following the resection of an AVM confined to the nasal cavity.

\section{Case report}

A 63-year-old male presented to the Department of Otolaryngology, Chi-Mei Medical Center (Tainan, Taiwan) in December 2011 with a history of nasal bleeding that had occurred intermittently for $\sim 5$ years. The patient also complained of blurred vision, particularly in the right eye. The symptoms were aggravated with time, and the patient visited the hospital for assistance. Sinoscopy showed that the cause of the bleeding was a tumor in the left nasal cavity. Magnetic resonance imaging and computed tomography scans showed soft-tissue mass lesions with post-contrast enhancement in the left nasal cavity, consistent with a vascular lesion, such as an AVM (Fig. 1A-C).

Angiography showed a large tangle of abnormal vascular channels in the nasal cavity, with a blood supply originating from the infraorbital and sphenopalatine branches of the left internal maxillary artery, the bilateral facial arteries and the left ophthalmic artery, and with early venous drainage into dilated and tortuous areas of the nasal mucosal vessels, bilateral facial veins (more in the right side), bilateral superior ophthalmic veins (more in the right side) and frontal scalp veins on the right side (Fig. 1D). AVM was highly suspected. Pre-operatively, the patient underwent superselective embolization, including occlusion of the bilateral sphenopalatine arteries, right descending palatine artery, a small branch of the left superficial temporal artery and the left facial artery.

The following day, the patient underwent a complete resection of the vascular tumor with sinoscopic assistance using a lateral rhinotomy approach (Fig. 2A). Subsequently, 

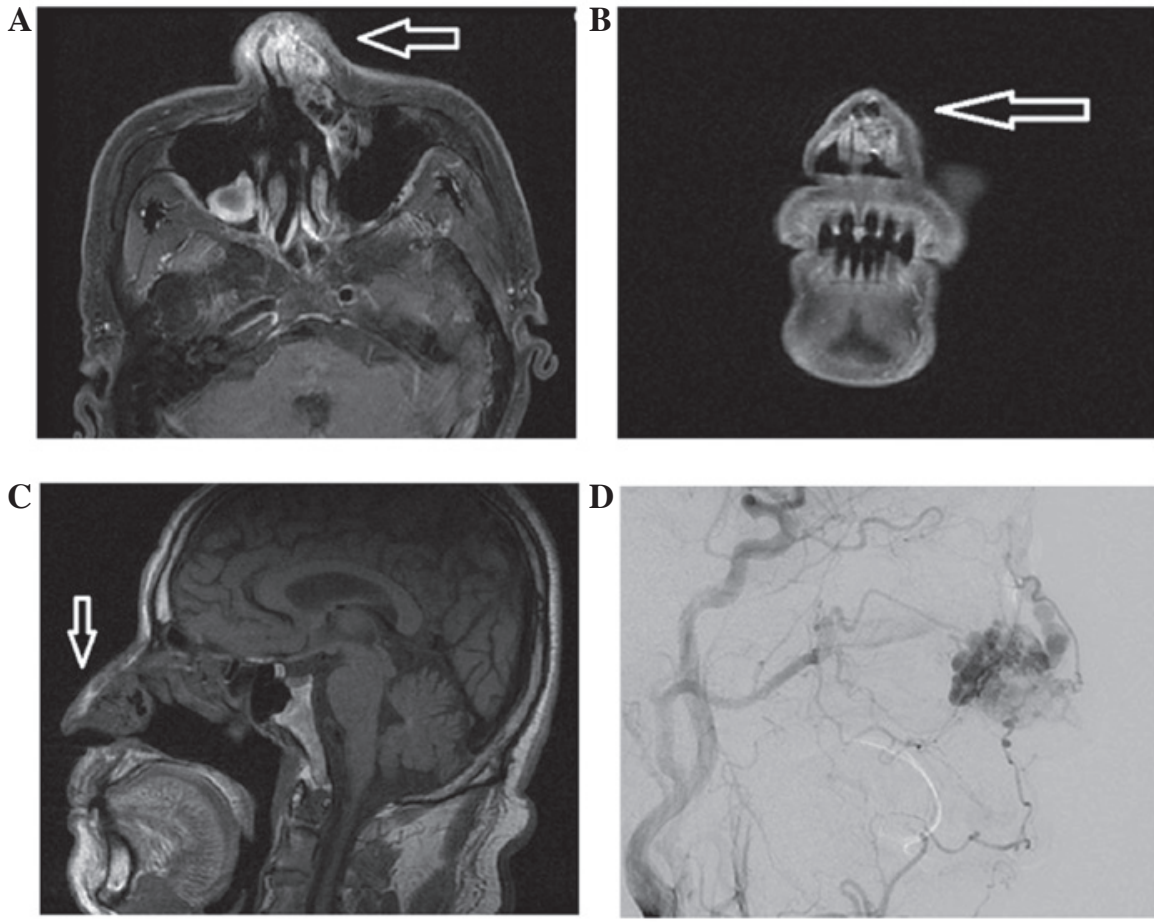

Figure 1. Magnetic resonance imaging scans showing (A-C) a soft tissue mass (arrows) with post-contrast enhancement in the nasal cavity. (D) Angiography showing a large abnormal vascular tangle in the nasal cavity.
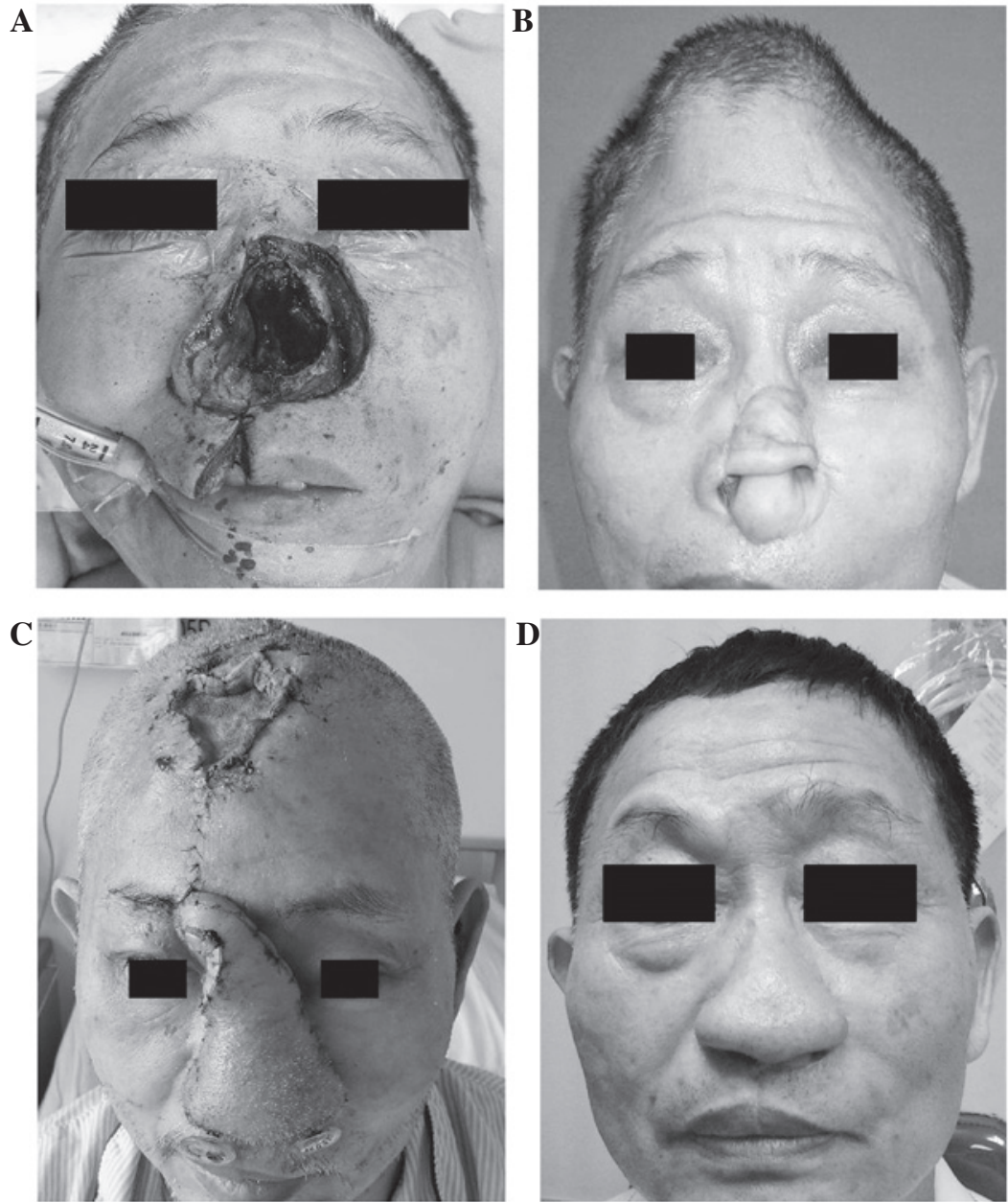

Figure 2. Images of the patient (A) after complete resection of the nasal cavity lesion, (B) after free radial forearm flap reconstruction and implantation of a forehead tissue expander and (C) after nasal reconstruction with a full-thickness paramedian forehead flap, a nasal dorsum, columella and upper lateral cartilage reconstruction with rib cartilage, and a nasal alar reconstruction with left ear cartilage. (D) The final result at 1.5 years after the initial surgery. 
nasal reconstruction using a radial forearm free flap and a split thickness skin graft was performed, due to the extensive involvement of the vascular tumor in the nasal area. Implantation of tissue expanders on the forehead was performed at the same time for later nasal reconstruction (Fig. 2B). Pathological examination confirmed an AVM. Post-operatively, the blurred vision subsided. Sequential nasal reconstruction surgeries, including a reconstruction using a full-thickness paramedian forehead flap, a reconstruction of the nasal dorsum, columella and upper lateral cartilage with rib cartilage graft, and a nasal alar reconstruction with left ear cartilage, were performed in April 2012 (Fig. 2C). Flap deviation was also performed in April, while flap debulking was performed in June 2012. During 1.5 years of follow-up, no recurrence was noted and the patient possessed an acceptable cosmetic appearance (Fig. 2D). The patient remains under follow-up and the wound has healed well.

\section{Discussion}

An AVM is a structural vascular abnormality in which arterial vessels connect with venous vessels without capillary connections. Progression may be due to stimuli, including pregnancy, trauma, infection and puberty, or iatrogenic injuries, including biopsy, proximal ligation and subtotal excision $(1,2)$. The abnormality is a high-flow vascular malformation with multiple low-resistance shunts $(2,3)$. In the present patient, angiography demonstrated a large tangle of abnormal vessels in the nasal cavity, with a blood supply originating from the infraorbital and sphenopalatine branches of the left internal maxillary artery, the bilateral facial arteries and the left ophthalmic arteries, and with early venous drainage into dilated and tortuous areas of the nasal mucosal vessels, bilateral facial veins (more in the right side), bilateral superior ophthalmic veins (more in the right side) and the right frontal scalp vein.

Clinically, the overlying skin of the AVM may appear normal, or as a pulsatile mass, red in color and with an increased local temperature $(3,4)$.

Other symptoms and signs may include pain, ulceration, excessive growth, profuse bleeding and even congestive heart failure $(3,4)$. In the present patient, constant nasal bleeding was the major complaint. The patient also experienced blurred vision, particularly in the right eye, which could have been associated with multiple shunts of the AVM involving the ophthalmic artery and vein (particularly on the right side). Following the complete resection of the nasal lesion, the blurred vision subsided, which strongly suggested that it was caused by the nasal AVM.

The Schobinger classification is one of the staging systems for AVM and includes the following stages: Stage I, blushing and warm cutaneous lesions; stage II, bruit, audible pulsations and expanding lesions; stage III, pain, ulceration, bleeding and infection; and stage IV, cardiac failure (5). The present patient exhibited stage III disease.

In the present study, pathological examination confirmed an AVM, however, occasionally, distinguishing between AVMs and hemangiomas is confusing. The use of elastic tissue stains is an useful ancillary tool to distinguish between these two conditions. The presence of arteries and arterioles is an integral feature of an AVM. The presence of intra-lesional nerves can also be useful to distinguish between AVMs and hemangiomas on hematoxylin and eosin-stained sections.

AVMs are mainly found in the intracranial region (6). Extracranial involvement is uncommon; the most common sites are the cheeks, ears, nose and forehead (in order of frequency in the extracranial region) (7). Other rare locations, such as the mandible, have also been reported (8). Following a search on PubMed (http://www.ncbi.nlm.nih.gov/pubmed), certain studies on nasal AVM were found; however, lesions confined only to the nasal cavity were rare, as was the resection of this type of defect. Only a few studies have reported the use of either local or free flaps alone for the reconstruction of AVM involving the nasal cavity following resection (1-5). One study described the use of a combination of free radial forearm and forehead flaps without prosthesis for nasal reconstruction in 2011 (9); however, for the management of nasal AVM, reconstruction using a combination of free radial forearm and local forehead flaps following resection of AVM confined to the nasal cavity has not been reported in the literature (1-6).

Pre-operatively deciding the extent of the resection of the AVM is crucial. Magnetic resonance imaging is used to determine the extent of the soft-tissue involvement, while computed tomography is able to delineate any bone involvement (10). Selective angiography is useful for the investigation of AVM, as it can identify specific vascular abnormalities and demonstrate the flow characteristics, feeding vessels and dangerous anastomoses (11).

Highly selective embolization as a single treatment modality is rarely successful with AVMs, due to the later development of novel vascular pathways. Embolization leads to a marked reduction in the blood flow within the vascular tumor, which decreases surgical blood loss and permits a complete resection of the tumor (11); however, pre-operative embolization should not be used to reduce the extent of resection (12). Partial resection is not curative and should be avoided, since revascularization and novel collateral circulation can lead to recurrence (7), and may encourage further shunting and reexpansion (12). Proximal artery ligation alone should also be avoided for the treatment of AVMs due to novel collateral circulation $(3,4)$.

A multidisciplinary approach is recommended for the management of an AVM (2). The combined use of a complete surgical excision and prior superselective embolization is the treatment of choice for AVMs $(10,11,13)$. It has been shown that it is best for the complete surgical resection to be performed within $48 \mathrm{~h}$ of highly selective embolization, as the resulting inflammation neutralizes hemodynamic benefits, making surgery more challenging (2-4). In the present patient, superselective embolization was performed first, then 1 day later, in order to avoid recurrence, a complete surgical resection of the lesions in the nasal cavity was performed. Due to the extensive involvement of the nasal AVM, the nasal defect was hard to reconstruct without the use of a flap. Reconstruction should include the internal nasal lining. Use of distant tissue through microvascular transfer is a way of bringing this lining to within the nose $(9,14,15)$. In the present study, the free radial forearm flap was used for the lining. The tissue expander was also placed in the forehead, and further nasal reconstruction was performed. During the surgeries, a full-thickness paramedian forehead flap was used. The nasal dorsum, columella 
and upper lateral cartilage area were reconstructed using rib cartilage graft. A nasal alar reconstruction was achieved using the left ear cartilage.

After 1.5 years of follow-up, no recurrence was noted in the present patient. The cosmetic results and olfactory perception of the patient were acceptable; however, rhinomanometry showed an increased inspiratory airflow resistance in the right naris compared with the left, which may have been due to the smaller nasal pathway through the right naris with greater air flow resistance.

In conclusion, AVM confined to the nasal cavity is considerably rare. Reconstruction using a combination of and local forehead flaps following resection of an AVM confined to the nasal cavity had not previously been reported in the literature. The present study reported a case of complete resection and reconstruction using a free radial forearm flap combined with a forehead flap, with acceptable cosmetic results. It should be noted that the differential diagnosis of blurred vision should include nasal diseases, such as AVM.

\section{References}

1. Weinzweig N, Chin G, Polley J, Charbel F, Shownkeen H and Debrun G: Arteriovenous malformation of the forehead, anterior scalp, and nasal dorsum. Plast Reconstr Surg 105: 2433-2439, 2000.

2. Bhandari PS, Sadhotra LP, Bhargava P, Bath AS, Mukherjee MK and Maurya S: Management strategy for facial arteriovenous malformations. Indian J Plast Surg 41: 183-189, 2008.

3. Kohout MP, Hansen M, Pribaz JJ and Mulliken JB: Arteriovenous malformations of the head and neck: Natural history and management. Plast Reconstr Surg 102: 643-654, 1998.
4. Pompa V, Valentini V, Pompa G, Di Carlo S and Bresadola L: Treatment of high flow arteriovenous malformations (AVMs) of the head and neck with embolization and surgical resection. Ann Ital Chir 82: 253-259, 2011.

5. Pompa V, Brauner E, Bresadola L, Di Carlo S, Valentini V and Pompa G: Treatment of facial vascular malformations with embolization and surgical resection. Eur Rev Med Pharmacol Sci 16: 407-413, 2012

6. Nocini PF, Fior A, Tolo C and Bertossi D: Arteriovenous Malformation of the nasal ala: A case report. J Oral Maxillofac Surg 58: 1303-1309, 2000.

7. Woo HJ, Song SY, Kim YD and Bai CH: Arteriovenous malformation of the external ear: A case report. Auris Nasus Larynx 35: 556-558, 2008.

8. Chhoeurn V, de Villa GH and Lo LJ: Osseous regeneration after embolization of mandibular arteriovenous malformation. Chang Gung Med J 26: 937-942, 2003.

9. Menick FJ and Salibian A: Microvascular repair of heminasal, subtotal, and total nasal defects with a folded radial forearm flap and a full-thickness forehead flap. Plast Reconstr Surg 127: 637-651, 2011.

10. Coskun BU, Sozen E, Basak T, Alkan S and Dadas B: Arteriovenous malformation of the nasopharynx: A case report. Int $\mathrm{J}$ Pediatr Otorhinolaryngol 69: 1287-1290, 2005.

11. Kim KS: Arteriovenous malformation in the pretragal region: Case report. Head Neck 33: 281-285, 2011.

12. Lowe LH, Marchant TC, Rivard DC and Scherbel AJ: Vascular malformations: Classification and terminology the radiologist needs to know. Semin Roentgenol 47: 106-117, 2012.

13. Nekooei S, Hosseini M, Nazemi $S$ and Talaei-Khoei $M$ : Embolization of arteriovenous malformation of the maxilla. Dentomaxillofacial Radiol 35: 451-455, 2006. Antunes MB and Chalian AA: Microvascular reconstruction of nasal defects. Facial Plast Surg Clin North Am 19: 157-162, 2011.

14. Antunes MB and Chalian AA: Microvascular reconstruction of nasal defects. Facial Plast Surg Clin North Am 19: 157-162, 2011.

15. Moore EJ, Strome SA, Kasperbauer JL, Sherris DA and Manning LA: Vascularized radial forearm free tissue for lining in nasal reconstruction. Laryngoscope 113: 2078-2085, 2003. 